\title{
Estimativa de valores corrigidos e o efeito da correção pelo erro de medida em dados dietéticos obtidos por Questionário de Frequência Alimentar para Adolescentes (QFAA)
}

\author{
Estimate of corrected values and the effect of correction \\ for measurement error in dietary data obtained \\ by the Food Frequency Questionnaire for Adolescents (AFFQ)
}

Silvia Maria Voci ${ }^{1}$

Carla Cristina Enes

Alexandre Romero ${ }^{1}$

Betzabeth Slater ${ }^{1}$

\begin{abstract}
The scope of this study was to estimate calibrated values for dietary data obtained by the Food Frequency Questionnaire for Adolescents (FFQA) and illustrate the effect of this approach on food consumption data. The adolescents were assessed on two occasions, with an average interval of twelve months. In 2004, 393 adolescents participated, and 289 were then reassessed in 2005. Dietary data obtained by the FFQA were calibrated using the regression coefficients estimated from the average of two 24-hour recalls (24HR) of the subsample. The calibrated values were similar to the the $24 \mathrm{HR}$ reference measurement in the subsample. In 2004 and 2005 a significant difference was observed between the average consumption levels of the FFQA before and after calibration for all nutrients. With the use of calibrated data the proportion of schoolchildren who had fiber intake below the recommended level increased. Therefore, it is seen that calibrated data can be used to obtain adjusted associations due to reclassification of subjects within the predetermined categories.
\end{abstract}

Key words Adolescent, Diet, Bias, Linear regression, Public health
Resumo O objetivo do estudo foi estimar valores corrigidos para os dados dietéticos obtidos por QFAA na amostra principal de uma coorte de adolescentes e ilustrar o efeito desta abordagem nas informações de consumo alimentar. Os adolescentes foram avaliados em dois momentos, com intervalo médio de 12 meses. Em 2004, participaram 393 escolares, sendo reavaliado, em 2005, um total de 289 adolescentes. Dados dietéticos obtidos por Questionário de Frequência Alimentar para Adolescentes (QFAA) foram corrigidos utilizando-se os coeficientes de regressão estimados a partir da média de dois Recordatórios de 24 horas (R24h) da subamostra. Os valores corrigidos se aproximaram dos obtidos por R24h na subamostra. Em 2004 e 2005 observou-se diferença significativa entre as médias de consumo do QFAA antes e após a calibração para todos os nutrientes. Com o uso dos dados corrigidos aumentou a proporção de escolares que apresentaram consumo de fibras abaixo do recomendado. Portanto, conclui-se que dados calibrados podem ser utilizados para a obtenção de associações corrigidas devido ao deslocamento dos indivíduos dentro de categorias pré-determinadas. Palavras-chave Adolescente, Dieta, Viés, Modelos lineares, Saúde pública

${ }^{1}$ Faculdade de Saúde Pública, Universidade de São Paulo. Av. Dr. Arnaldo 715, Cerqueira Cesar. 01246-904 São Paulo SP. smvoci@usp.br 


\section{Introdução}

Estudos epidemiológicos frequentemente utilizam dados autorrelatados de consumo alimentar para se aproximarem da ingestão dietética verdadeira. No entanto, os dados dietéticos são susceptíveis ao erro de medida, cuja magnitude e direção ainda são pouco conhecidas ${ }^{1}$.

Nas duas últimas décadas, o estudo do erro de medida favoreceu o surgimento de métodos de calibração paramétricos e não-paramétricos, utilizados para a correção dos valores dietéticos e, consequentemente, das medidas associativas obtidas em estudos epidemiológicos ${ }^{2-4}$.

O método estatístico da regressão de calibração, descrito primeiramente por Rosner et al. ${ }^{2}$, tem sido o mais utilizado, estimando o fator de correção ou de calibração $\lambda$, que representa a inclinação da reta de regressão. Essa reta é estimada a partir da regressão dos valores "verdadeiros" (representados por um método de referência, geralmente Recordatórios de 24 horas ou Registros Alimentares), contra os valores obtidos por meio do Questionário de Frequência Alimentar (QFA) ${ }^{5}$. Esta abordagem tem propiciado a eliminação ou a redução do viés contido no instrumento testado (QFA), aproximando os novos valores aos obtidos por um método de referência ${ }^{6}$.

Poucos estudos de calibração de QFA foram publicados no Brasil até o momento. Em 2007, Slater et al. ${ }^{7}$ testaram a proposta metodológica da calibração em um banco de dados secundário. Mais recentemente, Voci et al. ${ }^{8}$ apresentaram os resultados de um estudo de calibração do QFAA, planejado e conduzido especificamente para esta finalidade, fornecendo os coeficientes específicos para a correção dos dados dietéticos de adolescentes de uma coorte de Piracicaba. De acordo com Hjartaker et al. ${ }^{9}$, embora a proposta de estudos metodológicos seja a de fornecer dados mais acurados de consumo alimentar, geralmente seus resultados não são implementados nas análises principais da relação entre dieta e doença.

Considerando-se estes aspectos, o objetivo do presente estudo foi estimar valores corrigidos para os dados dietéticos obtidos por QFAA na amostra principal de uma coorte de adolescentes de Piracicaba-SP e ilustrar o efeito desta abordagem nos dados de consumo alimentar.

\section{Métodos}

Esse trabalho foi desenvolvido utilizando-se os dados do estudo intitulado "Consumo dietético e atividade física como determinantes das mudanças do índice de massa corporal de uma coorte de adolescentes matriculados na rede pública de ensino da cidade de Piracicaba, São Paulo", financiado pela Fundação de Amparo à Pesquisa do Estado de São Paulo.

O processo de amostragem da coorte foi o de múltiplos estágios e considerou informações sobre a prevalência de excesso de peso obtida em estudos anteriores realizados com população semelhante no país. Também foram considerados erro tipo I de 5\% e erro tipo II de $20 \%$. A amostragem foi iniciada pela ordenação das escolas de acordo com as regiões do município. Foram sorteadas doze escolas, considerando que onze estavam localizadas em zonas urbanas e uma em zona rural. Uma das escolas urbanas foi retirada da amostra representativa para a condução do subestudo de calibração do QFAA.

As classes (séries) das escolas foram sorteadas por amostragem aleatória simples e, em cada uma delas, realizou-se o sorteio de determinado número de alunos, levando-se em consideração o número total de alunos da escola.

Os adolescentes foram entrevistados em dois momentos, respeitando-se o intervalo médio de doze meses entre a primeira e a segunda entrevista. Na primeira avaliação foram obtidas as medidas antropométricas (peso e altura), informações relativas ao consumo alimentar habitual, à atividade física e à maturação sexual dos adolescentes, bem como as variáveis demográficas e as socioeconômicas. No segundo momento, os alunos foram reavaliados, sendo novamente coletados dados de antropometria, consumo alimentar, atividade física e maturação sexual. O enfoque do estudo está nos dados de consumo alimentar obtidos a partir do QFAA, desenvolvido e validado por Slater et al. ${ }^{10}$.

Para as análises foram incluídos dados de adolescentes com idade entre 10 e 16 anos, de ambos os momentos da coorte. Em razão das perdas de acompanhamento inerentes aos estudos de coorte, foram analisados dados de 393 adolescentes que participaram do estudo em 2004 e 289 que participaram em 2005, o que representa uma perda de $26,6 \%$ da amostra inicial. Foram excluídos os indivíduos que apresentaram dados incompletos, consumo diário de energia inferior a $500 \mathrm{kcal}$ ou superior a $7000 \mathrm{kcal}^{11}$.

Deve-se ressaltar que os dados de 2004 e de 2005 foram analisados separadamente, ou seja, de forma transversal.

O QFAA incluiu perguntas relativas à frequência usual de consumo de 94 itens alimentares 
referentes a um período de seis meses. As porções utilizadas neste instrumento representavam o consumo médio, em gramas, de cada item alimentar ${ }^{10}$.

O instrumento contou com sete opções de frequência de consumo, a saber: nunca; menos de uma vez ao mês; de 1 a 3 vezes ao mês; 1 vez por semana; de 2 a 4 vezes por semana; 1 vez ao dia; e, 2 ou mais vezes ao dia. Visando obter respostas fidedignas dos entrevistados, utilizou-se um material fotográfico elaborado especialmente para este estudo, apresentando diferentes utensílios comumente utilizados na alimentação tais como xícaras, pratos, copos e talheres.

\section{Correção dos dados dietéticos}

Para estimar os coeficientes necessários para a calibração, inicialmente se partiu do pressuposto de que o $\lambda$ (inclinação da reta estimada) obtido a partir da utilização de valores de referência $(R)$ seria igual ao obtido se fossem utilizados os valores verdadeiros de ingestão habitual (desconhecido, $T)^{6}$.

$$
\mathrm{E}(R \mid Q)=\mathrm{E}(T \mid Q)
$$

Neste sentido, foi conduzido um estudo de calibração em uma subamostra, retirada da coorte analisada no presente trabalho, possibilitando a aplicação de um método de referência em mais de um momento de avaliação para obter dados da dieta habitual ${ }^{8}$. No estudo de calibração, foram estimados os coeficientes da reta de regressão (Apêndice) para a correção de valores dietéticos, a partir da análise de dados de 74 adolescentes, de ambos os sexos, considerando-se como referência ( $R$ ) a média de dois $\mathrm{R} 24 \mathrm{~h}$. Na regressão linear, o valor de referência foi considerado como a variável dependente e o valor obtido por QFAA como a variável independente.

A digitação das informações do QFAA foi realizada utilizando-se o software Dietsys, versão 4.01, procedendo-se à dupla digitação para eliminação de eventuais erros de digitação. Os dados do R24h da subamostra foram digitados no software $\mathrm{Nu}$ trition Data System for Research (NDS).

Os coeficientes da reta utilizados para a calibração foram os obtidos para energia (kcal), macronutrientes $(\mathrm{g})$, fibra $(\mathrm{g})$, colesterol $(\mathrm{mg})$, cálcio $(\mathrm{mg})$, vitamina $\mathrm{C}(\mathrm{mg})$ e retinol $(\mu \mathrm{g})$.

\section{Análises estatísticas}

Todas as variáveis quantitativas estudadas apresentaram distribuição normal. Realizaramse análises descritivas e as variáveis dietéticas que se apresentaram fortemente correlacionadas com a energia foram ajustadas por esta variável de acordo com o método dos resíduos, descrito por Willett $^{12}$. O ajuste foi realizado para macronutrientes e cálcio (em ambos os momentos) e para a fibra (seguimento).

Para estimar os valores corrigidos de cada variável dietética da coorte (dados de consumo obtidos na linha de base e no seguimento) aplicou-se modelo de regressão linear simples, utilizando-se os coeficientes (intercepto $v$ e inclinação da reta $\lambda$ ) encontrados no subestudo de calibração publicado por Voci et al. $^{8}$ e apresentados na Tabela 1. Na equação de regressão apresentada a seguir, os valores de $\hat{y}$ se referem aos novos valores obtidos para cada nutriente, representando os dados do questionário de frequência alimentar corrigidos na coorte.

$$
\mathrm{E}(\hat{Y} \mid Q)=\hat{\mathrm{v}}+\hat{\lambda} Q
$$

As médias do QFAA corrigido foram comparadas às médias do QFAA ajustado pela energia utilizando-se teste t de Student pareado. Adotou-se nível de significância de $5 \%$.

Para mostrar o efeito da calibração nos dados dietéticos, procedeu-se à classificação do consumo dos adolescentes antes e depois desta abordagem. Para tanto, utilizaram-se pontos de corte baseados nas DRIs para energia (Estima-

Tabela 1. Coeficientes da regressão de calibração para as variáveis dietéticas do estudo de Voci ${ }^{8}$.

\begin{tabular}{lrrrr}
\hline $\begin{array}{l}\text { Variável } \\
\text { Dietética }\end{array}$ & $\boldsymbol{v}$ & \multicolumn{1}{c}{ IC95\% } & $\boldsymbol{\lambda}$ & \multicolumn{1}{c}{ IC95\% } \\
\hline Energia & 1532,45 & 984,$90 ; 2080,00$ & $0,25^{*}$ & 0,$06 ; 0,44$ \\
Proteína & 60,07 & 37,$54 ; 82,60$ & 0,22 & $-0,04 ; 0,48$ \\
Lipídio & 57,72 & 34,$21 ; 81,22$ & $0,26^{*}$ & 0,$03 ; 0,49$ \\
Carboidrato & 244,97 & 153,$01 ; 336,93$ & 0,12 & $-0,11 ; 0,36$ \\
Fibra & 12,32 & 6,$41 ; 18,23$ & $0,32^{*}$ & 0,$06 ; 0,57$ \\
Colesterol & 142,24 & 62,$47 ; 222,02$ & 0,24 & $-0,08 ; 0,55$ \\
Cálcio & 551,71 & 297,$00 ; 806,42$ & 0,18 & $-0,16 ; 0,52$ \\
Vitamina C & $-2,48$ & $-36,66 ; 31,71$ & $0,40^{*}$ & 0,$19 ; 0,60$ \\
Retinol & 156,49 & 84,$77 ; 228,20$ & $0,17^{*}$ & 0,$03 ; 0,31$ \\
\hline
\end{tabular}

v: intercepto da equação de regressão de calibração. $\lambda$ : coeficiente de calibração obtido com variáveis dietéticas ajustadas pela energia. * nível de significância $<0,05$ 
ted Energy Requirement - EER), proteína (Estimated Average Requirement - EAR), carboidrato (EAR), cálcio (Adequate Intakes - AI), fibra (AI), vitamina C (Recommended Dietary Allowances - RDA) e retinol (EAR) ${ }^{13}$.

Este estudo foi aprovado pelo Comitê de Ética em Pesquisa da Faculdade de Saúde Pública da USP. Foram entrevistados somente aqueles que apresentaram o termo de consentimento livre e esclarecido assinado pelos pais ou responsáveis.

\section{Resultados}

$\mathrm{Na}$ ocasião da linha de base foram entrevistados 393 adolescentes, sendo $46 \%$ do sexo masculino. A média de idade foi de 12,4 anos (desvio-padrão de 1,51 anos). Cerca de 20\% dos adolescentes foram classificados como pré-púberes segundo estágios de maturação sexual. Em relação ao estado nutricional, observou-se que $21 \%$ apresentaram excesso de peso (10\% sobrepeso e $11 \%$ obesidade).

$\mathrm{Na}$ segunda avaliação (seguimento), 289 adolescentes foram estudados, sendo que destes, $44 \%$ eram do sexo masculino. Em relação à maturação sexual, 8\% dos adolescentes foram classificados como pré-púberes e a média de idade foi de 12,8 anos (desvio-padrão de 1,34 anos). A prevalência de excesso de peso encontrada foi de $28 \%$, distribuídos em igual proporção para sobrepeso e obesidade. Verificou-se maior proporção de excesso de peso entre os meninos (30\%) comparados às meninas (25\%).

$\mathrm{O}$ intervalo entre a primeira e a segunda entrevistas variou de 301 a 445 dias, sendo o intervalo médio de 362 dias (desvio-padrão de 33 dias). Cinquenta e dois por cento dos adolescentes que não participaram do segundo momento do estudo (perdas) eram do sexo feminino. Os principais motivos que levaram às perdas foram a mudança de escola, de endereço ou de telefone, razões que dificultaram a localização dos participantes.

$\mathrm{Na}$ Tabela 2 estão apresentados os valores médios de consumo para energia e nutrientes obtidos nos dois momentos da coorte, antes e após a calibração. A título de ilustração também são apresentados os valores de ingestão já publicados por Voci et al. ${ }^{8}$ para o R24h da subamostra, utilizado como referência.

Para o carboidrato, por exemplo, observouse que as médias do QFAA se distanciavam das médias do R24h. Após a calibração, esta diferença reduziu, verificando-se esta tendência de aproximação aos valores de referência para todos os nutrientes estudados. Por outro lado, o nutriente que obteve menor ajuste foi a vitamina $\mathrm{C}$, permanecendo superestimada em relação ao valor de referência da subamostra.

Ao se comparar as médias dos valores do QFAA antes e após a calibração verificou-se uma diferença estatisticamente significativa para todas as variáveis.

Na Tabela 3 está ilustrado o efeito da correção do erro de medida na classificação do consumo acima ou abaixo do ponto de corte utilizado. Foram observadas mudanças mais acentuadas para a fibra, vitamina $\mathrm{C}$ e retinol, após a calibração dos dados. Deve-se ressaltar o aumento da proporção de adolescentes que apresentaram um consumo de fibras abaixo do recomendado após a calibração tanto na linha de base quanto no seguimento. Situação semelhante ocorreu com o retinol, que após a calibração praticamente a totalidade (99\%) dos adolescentes revelou um baixo consumo desse nutriente. Ressalta-se que os dados referentes ao lipídio e colesterol não foram ilustrados nesta tabela, devido ao fato desses nutrientes não apresentarem valores recomendados (DRI) para serem utilizados como pontos de corte.

Em relação à energia (Figura 1), verificou-se que após a calibração, na linha de base, houve um aumento expressivo do percentual de indivíduos que apresentaram um consumo dentro do intervalo esperado de EER, passando de $5 \%$ para $69 \%$ e de $83 \%$ para $9 \%$ de adolescentes com consumo superior a EER.

\section{Discussão}

Esse trabalho teve como propósito estimar valores corrigidos para os dados dietéticos obtidos por QFAA (energia, macronutrientes, fibra, cálcio, colesterol, vitamina $\mathrm{C}$ e retinol) e ilustrar o efeito da calibração nos dados de consumo alimentar de adolescentes de uma coorte de Piracicaba.

São poucos os estudos publicados utilizando dados dietéticos corrigidos e, dentre os publicados, não são apresentados valores descritivos de ingestão antes e após a aplicação desta abordagem metodológica, dificultando a discussão dos resultados obtidos no presente estudo sobre o efeito da correção.

Deve-se ressaltar que os dados dietéticos corrigidos, obtidos por QFAA, são valores estimados a partir de regressão linear, capaz de minimizar o erro de medida, aproximando os valores preditos daqueles considerados de referência caso esti- 
Tabela 2. Média e desvio-padrão (DP) para energia e nutrientes obtidos por QFAA na coorte, antes e após a calibração, e valores de R24h obtidos na subamostra. Piracicaba, 2004 e 2005.

\begin{tabular}{|c|c|c|c|c|c|c|}
\hline \multirow{3}{*}{$\begin{array}{l}\text { Variável } \\
\text { Dietética }\end{array}$} & Linha de $b$ & e $\quad \mathrm{N}=393$ & Seguimento & $\mathrm{N}=289$ & Subamostra & $\mathrm{N}=74$ \\
\hline & \multicolumn{2}{|c|}{ QFAA } & \multicolumn{2}{|c|}{ QFAA } & \multicolumn{2}{|c|}{$\mathrm{R} 24 \mathrm{~h}$} \\
\hline & Média & DP & Média & DP & Média & DP \\
\hline \multicolumn{7}{|l|}{ Energia (kcal) } \\
\hline bruta & 3556,2 & 1343,4 & 3375,6 & 1344,3 & 2216,3 & 822,3 \\
\hline calibrada & 2421,5 & 335,8 & 2376,3 & 336,1 & - & - \\
\hline \multicolumn{7}{|l|}{ Proteína (g) } \\
\hline ajustada & 111,7 & 21,2 & 106,9 & 20,2 & 79,1 & 15,4 \\
\hline calibrada & 84,7 & 4,7 & 83,6 & 4,4 & - & - \\
\hline \multicolumn{7}{|l|}{ Lipídio (g) } \\
\hline ajustado & 135,2 & 20,4 & 122,5 & 18,5 & 84,0 & 13,5 \\
\hline calibrado & 92,9 & 5,3 & 89,6 & 4,9 & - & - \\
\hline \multicolumn{7}{|c|}{ Carboidrato (g) } \\
\hline ajustado & 502,1 & 54,3 & 483,7 & 49,1 & 292,2 & 35,9 \\
\hline calibrado & 305,2 & 6,5 & 303,0 & 5,9 & - & - \\
\hline \multicolumn{7}{|l|}{ Fibra (g) } \\
\hline ajustada & 30,1 & 11,3 & 27,5 & 6,7 & 19,4 & 6,5 \\
\hline calibrada & 21,9 & 3,6 & 21,1 & 2,2 & - & - \\
\hline \multicolumn{7}{|c|}{ Colesterol (mg) } \\
\hline bruto $^{*}$ & 339,4 & 156,9 & 312,9 & 172,3 & 200,6 & 84,5 \\
\hline calibrado & 223,7 & 37,7 & 217,4 & 41,4 & - & - \\
\hline \multicolumn{7}{|l|}{ Cálcio (mg) } \\
\hline ajustado & 970,5 & 205,0 & 878,7 & 196,3 & 681,4 & 209,6 \\
\hline calibrado & 726,4 & 36,9 & 709,9 & 35,3 & - & - \\
\hline \multicolumn{7}{|c|}{ Vitamina C (mg) } \\
\hline bruta* & 197,7 & 115,2 & 194,3 & 134,6 & 56,2 & 66,7 \\
\hline calibrada & 76,6 & 46,1 & 75,3 & 53,8 & - & - \\
\hline \multicolumn{7}{|l|}{ Retinol ( $\mu \mathrm{g})$} \\
\hline bruto & 631,6 & 346,7 & 584,3 & 316,5 & 239,1 & 124,6 \\
\hline calibrado & 263,9 & 59,0 & 255,8 & 53,8 & - & - \\
\hline
\end{tabular}

* Os valores de colesterol, vitamina C e retinol não foram ajustados pela energia antes da calibração. OBS.: Todas as variáveis dietéticas apresentaram médias estatisticamente diferentes para valores ajustados ou brutos e calibrados na linha de base e seguimento.

vessem disponíveis para os indivíduos da coorte. Quando observados estes valores corrigidos, nota-se que o QFAA inicialmente superestimou o consumo de todos os nutrientes e de energia.

Foram comparados os resultados descritivos corrigidos de ingestão com os dados de estudos conduzidos com adolescentes e que utilizaram métodos de referência para a avaliação do consumo. Para a energia e os macronutrientes a ingestão observada foi superior aos valores obtidos por Lee et al. ${ }^{14}$ e por Slater et al. ${ }^{7}$, e inferior aos apresentados por Stockman et al. ${ }^{15}$ e Albano e Souza ${ }^{16}$.

Para a fibra, o colesterol e o cálcio os valores corrigidos de ambos os momentos do presente estudo foram bastante superiores aos descritos por Lee et al. ${ }^{14}$. O valor médio de consumo de fibra encontrado foi superior ao valor equiva- lente ao percentil 99 descrito por Schenkel et al. ${ }^{17}$. Para o cálcio, a média de consumo da linha de base na coorte estudada foi semelhante à mediana apresentada pelos mesmos autores.

Em relação à vitamina $\mathrm{C}$, observou-se que sua ingestão média foi semelhante à mediana de consumo de 75,8 mg na amostra de Schenkel et al. ${ }^{17}$. Quanto ao retinol, os valores médios encontrados foram próximos aos classificados no percentil 10 do mesmo estudo.

Quando observados os resultados categorizados usando-se as DRI como ponto de corte para o carboidrato, não foram identificados indivíduos com ingestão abaixo dos valores de EAR em ambos os momentos, sendo este fato concordante com resultados recentes e que apontam um elevado consumo de açúcares ${ }^{14,18}$. 
Verificou-se que todos os adolescentes apresentaram consumo de cálcio inferior aos valores de AI segundo o sexo. Reconhecidamente o cálcio é um nutriente que dificilmente tem suas necessidades atingidas, especialmente neste grupo

Tabela 3. Proporção (\%) de adolescentes categorizados a partir de valores de EAR, antes e depois da calibração dos dados de ingestão de nutrientes obtidos em duas avaliações da coorte. Piracicaba (SP), 2004 e 2005.

\begin{tabular}{cccccc}
\hline Variável & \multicolumn{2}{c}{2004} & & \multicolumn{2}{c}{2005} \\
\cline { 2 - 3 } \cline { 5 - 6 } Dietética & Ajustado* & Calibrado & & Ajustado* & Calibrado \\
\hline Proteína & & & & & \\
Abaixo & 0 & 0 & & 0 & 0 \\
Acima & 100 & 100 & & 100 & 100 \\
Carboidrato & & & & 0 & 0 \\
Abaixo & 0 & 0 & & 0 & 100 \\
Acima & 100 & 100 & & 100 & \\
Fibra & & & & & \\
Abaixo & 52 & 92 & & 59 & 98 \\
Acima & 48 & 8 & & 41 & 2 \\
Cálcio & & & & 97 & 100 \\
Abaixo & 95 & 100 & & 97 & 0 \\
Acima & 5 & 0 & & 3 & \\
Vitamina C & & & & & \\
Abaixo & 4 & 22 & & 3 & 30 \\
Acima & 96 & 78 & & 97 & 70 \\
Retinol & & & & & \\
Abaixo & 36 & 99 & & 42 & 99 \\
Acima & 64 & 1 & 58 & 1 \\
\hline
\end{tabular}

* Os valores de vitamina $\mathrm{C}$ e retinol não foram ajustados pela energia antes da calibração. populacional. Estudos conduzidos por Lee et al. ${ }^{14}$ e Nielson e Popkin ${ }^{19}$ apontaram para um aumento do consumo de refrigerantes e de bebidas carbonatadas, com adição de açúcar, em detrimento do consumo de leite, o que poderia explicar estes valores de ingestão que vêm sendo descritos na literatura nas últimas décadas.

Deve-se ressaltar que os valores de DRIs foram utilizados somente como referência para a categorização dos indivíduos, não sendo possível discutir sobre prevalência de inadequação, uma vez que se trata de valores obtidos por QFA.

Como mencionado anteriormente, são poucos os estudos que apresentam resultados corrigidos e que ilustrem os efeitos desta abordagem. Alguns estudos internacionais publicados descrevem a utilização do método de calibração diretamente na correção da medida associativa obtida na amostra principal, e outros, no entanto, aplicam as equações para a correção pelo erro de medida diretamente nos dados dietéticos obtidos na coorte, como realizado no presente estudo.

Gonzalez et al..$^{20}$ estudaram a relação entre o risco de câncer gástrico e de adenocarcinoma esofágico e o consumo de carnes, utilizando dados do European Prospective Investigation Into Cancer and Nutrition (EPIC). No trabalho citado, os autores utilizaram dados dietéticos corrigidos, estimados para cada indivíduo da coorte, para a obtenção de Harzard Ratio. Com base nos resultados encontrados pelos autores, notase que de maneira geral as medidas associativas aumentaram com a correção e os intervalos de confiança se ampliaram. Somente a relação entre

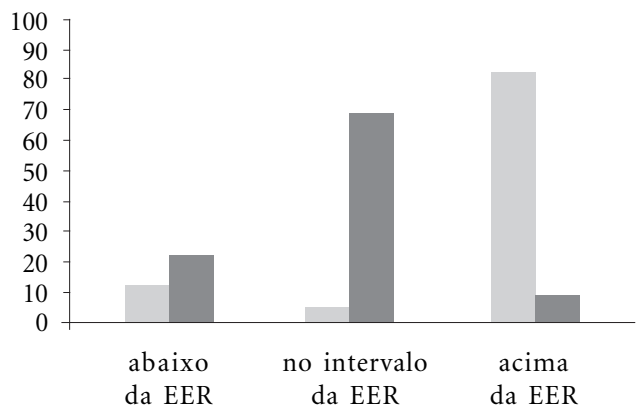

2004

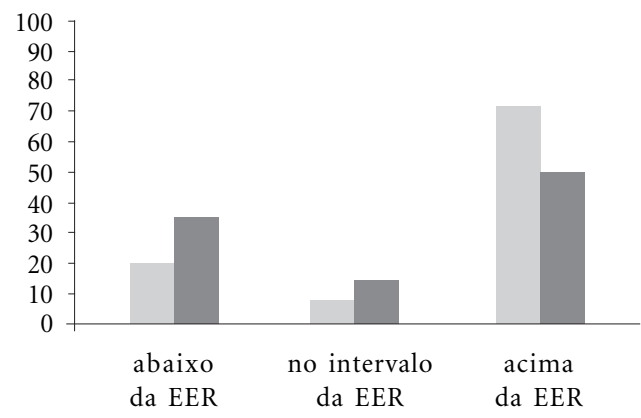

2005

$$
\text { antes depois }
$$

antes

depois

Figura 1. Proporção (\%) de adolescentes categorizados a partir de valores de EER, antes e depois da calibração dos dados de ingestão de energia. Piracicaba (SP), 2004 e 2005. 
o consumo de carnes processadas e o câncer gástrico tornou-se significativa após a correção.

Segundo Jain et al. ${ }^{21}$, após a correção pelo erro de medida da variável de exposição, é possível que a medida associativa se afaste da homogeneidade quando comparada à outra não corrigida, podendo evidenciar uma relação atenuada. No entanto, estas estimativas também poderão apresentar menor precisão, uma vez que há a possibilidade de ampliação do IC95\%.

Outro exemplo do efeito da calibração é citado por Thompson et al. ${ }^{22}$. Quando se obtém um fator de atenuação mais próximo de 1,0 (denotando menor erro de medida na variável de exposição), a correção que será aplicada diretamente à medida associativa será menor. De acordo com o exemplo dado pelos mesmos autores, se um risco relativo de 2,0 para uma determinada mudança na exposição for ajustado por um fator de atenuação de 0,5 , a medida associativa corrigida será igual a 1,41 , enquanto que se o fator de atenuação for igual a 0,1 (denotando maior erro de mensuração na variável de exposição), o risco relativo corrigido será de 1,07.

Bingham et al. ${ }^{23}$ obtiveram medidas associativas aplicando o método de calibração aos dados dietéticos, assim como no presente estudo. Para a correção dos dados foi utilizada equação multivariada, levando em consideração o consumo de fibra dietética, energia proveniente de gorduras e de fontes não lipídicas, simultaneamente. Para cada indivíduo da coorte foram obtidos valores corrigidos aplicando-se o intercepto e as inclinações das variáveis das equações específicas para sexo e centro.

A exemplo do que já foi descrito por outros autores ${ }^{3,4,6-8}$, nota-se a diminuição dos desviospadrão para os valores corrigidos, o que provavelmente se deve à não adesão de valores extremos ao pressuposto de linearidade entre os métodos de avaliação do consumo alimentar ${ }^{6}$.

Segundo Ferrari et al. ${ }^{24}$ a regressão de calibração concentra os valores corrigidos da exposição acerca da média de referência como uma consequência do encurtamento na distribuição dos valores preditos. Nesse estudo ${ }^{24}$, a análise dos componentes da variância para os valores preditos de ingestão (corrigidos) mostrou forte encurtamento em direção aos valores médios, de forma que as estimativas dos coeficientes de correlação intraclasse (CCI), de valores corrigidos, foram duas vezes superiores às medidas do questionário bruto.

A vantagem da utilização de dados corrigidos em substituição aos brutos nas estimativas da relação entre dieta e doença foi apresentada por Fra- ser e Stram $^{25}$. Ferrari et al. ${ }^{24}$ também relatam a aplicação de dados dietéticos corrigidos e as mudanças nas "Hazard Ratios", atenuando a associação encontrada e reforçando o efeito protetor do consumo de peixes em países europeus, em relação ao desenvolvimento de câncer colo-retal.

Observando-se os resultados apresentados, é possível notar que os valores corrigidos na coorte se distanciam dos valores brutos e tornamse mais próximos aos observados nos R24h da subamostra, indicando uma possível redução do erro de medida. No entanto, é preciso ressaltar que a proposta da calibração linear apresenta fragilidades relacionadas aos seus pressupostos teóricos, não atingindo, por exemplo, valores discrepantes entre os métodos utilizados para levantar dados de consumo alimentar.

A regressão de calibração tem uma justificativa teórica baseada em uma aproximação linear com pressupostos de não correlação de erros de medidas entre R24h e QFA. A correlação entre os erros em medidas dietéticas autorreferidas provavelmente existe como uma consequência da tendência dos indivíduos de subestimar ou superestimar consistentemente seu consumo alimentar ${ }^{26-28}$. Nos casos univariados, em que se calibra a relação entre uma exposição dietética e o risco de doença, os fatores de atenuação podem ser superestimados se não houver a independência entre erros de medidas dos dois instrumentos, resultando em uma correção mode$\operatorname{rada}^{24}$.

A calibração linear assume que a medida obtida pelo método de referência fornece uma estimativa imparcial da exposição verdadeira. Segundo Ferrari et al. ${ }^{24}$, o erro relacionado à ingestão não está aleatoriamente distribuído e é provável que este esteja relacionado às características dos participantes do estudo, consequentemente fornecendo evidências na limitação do uso de R24h como medidas de referência.

No entanto, na falta de um "padrão ouro" para a avaliação do consumo alimentar, a média de inúmeras aplicações de recordatórios de $24 \mathrm{~h}$ ou de diários alimentares continua sendo utilizada como referência, mesmo que evidências tenham mostrado que esses métodos também são imperfeitos, com erros sistemáticos específicos dos indivíduos ${ }^{5,12}$.

Ressalta-se a necessidade de se continuar pesquisando abordagens mais recentes, como as não-paramétricas e as semi-paramétricas, que não exigem pressupostos de linearidade e normalidade ${ }^{3}$. A utilização de um modelo de correção de erro de medida inadequado ao compor- 
tamento do dado e, consequentemente, as violações às pressuposições do método podem acarretar problemas importantes como o surgimento de vieses adicionais ${ }^{5}$.

A incorporação de variáveis que ajustem o modelo de calibração, tais como a idade, os dias da semana, as estações do ano, dentre outros, a exemplo do que já vem sendo realizado no European Prospective Investigation into Cancer and Nutrition - EPIC $^{24}$, também deve ser considerada, bem como a necessidade de aprimoramento dos instrumentos já existentes no sentido de melhorar a acurácia dos dados dietéticos obtidos, atendendo a finalidades e populações específicas.
Salienta-se ainda que a correção de dados dietéticos pode impor mudanças importantes nos resultados de estudos que pautem suas análises em informações de consumo alimentar e, consequentemente, interferir na definição de políticas de alimentação e nutrição.

Concluímos que a correção dos dados dietéticos pelo erro de medida teve um efeito significativo, uma vez que os valores corrigidos diferiram estatisticamente dos dados brutos e interferiram na classificação dos indivíduos quando utilizados pontos de corte baseados nas DRI, ressaltando a importância de se utilizar estes dados corrigidos nas futuras análises desta coorte.

\section{Colaboradores}

SM Voci e CC Enes participaram da coleta de dados, análises estatísticas, redação e revisão do manuscrito; A Romero participou da coleta e análise de dados referentes à atividade física, e da revisão do manuscrito; e, B Slater foi a coordenadora do estudo principal, e participou da revisão do manuscrito.

\section{Referências}

1. Michels KB, Bingham SA, Luben R, Welch AA, Day NE. The Effect of Correlated Measurement Error in Multivariate Models of Diet. Am J Epidemiol 2004; 160(1):59-67.

2. Rosner B, Willett WC, Spiegelman D. Correction of logistic regression relative risk estimates and confidence intervals for systematic within-person measurement error. Stat Med 1989; 8(9):1051-1069.

3. Kaaks R, Riboli E, van Staveren W. Calibration of dietary intake measurements in prospective cohort studies. Am J Epidemiol 1995; 142(5):548-556.

4. Hoffmann K, Kroke A, Klipstein-Grobusch K, Boeing $\mathrm{H}$. Standardization of dietary intake measurements by nonlinear calibration using short-term reference data. Am J Epidemiol 2002; 156(9):862-870.

5. Kipnis V, Carroll RJ, Freedman LS, Li L. Implications of a new dietary measurement error model for estimation of relative risk: application to four calibration studies. Am J Epidemiol 1999; 150(6):642651.

6. Kynast-Wolf G, Becker N, Kroke A, Brandstetter BR, Wahrendorf J, Boeing H. Linear regression calibration: theoretical framework and empirical results in EPIC, Germany. Ann Nutr Metab 2002; 46(1):2-8.

7. Slater B, Marchioni DML, Voci SM. Aplicação de regressão linear para correção de dados dietéticos. Rev Saude Publica 2007; 41(2):190-196.

8. Voci SM, Slater B, Silva MV, Marchioni DML, Latorre MRDO. Estudo de calibração do Questionário de Frequência Alimentar para Adolescentes QFAA. Cien Saude Colet 2008; 16(4):2335-2343.

9. Hjartaker A, Andersen LF, Lund E. Comparison of diet measures from a food-frequency questionnaire with measures from repeated 24-hour recalls. The Norwegian Women and Cancer Study. Publ Health Nutr 2007; 10(10):1094-1103. 
10. Slater B, Philippi ST, Fisberg RM, Latorre MRDO. Validation of a semi-quantitative adolescent food frequency questionnaire applied at a public school in São Paulo, Brazil. Eur J Clin Nutr 2003; 57(5):629635.

11. Ludwig DS, Peterson KE, Gortmaker SL. Relation between consumption of sugar-sweetened drinks and childhood obesity: a prospective, observational analysis. Lancet 2001; 357(9255):505-508.

12. Willett WC. Nutritional Epidemiology. $2^{\text {nd }}$ ed. New York: Oxford University Press; 1998.

13. Institute of Medicine, Food and Nutrition Board. Dietary Reference Intakes. The Essential Guide to nutrient requirements. Washington: National Academy Press; 2006.

14. Lee SK, Novotny R, Daida YG, Vijayadeva, Gittelsohn J. Dietary patterns of adolescent girls in Hawaiii over a 2 year-period. J Am Diet Assoc 2007(6); 107:956-961.

15. Stockman NKA, Schenkel TC, Brown JN, Duncan AM. Comparison of energy and nutrient intakes among meals and snacks of adolescent males. Preventive Medicine 2005; 41(1):203-210.

16. Albano RD, Souza SB. Ingestão de energia e nutrientes por adolescentes de uma escola pública. $J$ Pediatr 2001; 77(6):512-516.

17. Schenkel TC, Stockman NKA, Brown JN, Duncan AM. Evaluation of energy, nutrient and dietary fiber intake of adolescent males. J Am Coll Nutr 2007; 26(3):264-271.

18. Toral N, Slater B, Silva MV. Consumo alimentar e excesso de peso de adolescentes de Piracicaba, São Paulo. Rev Nutr 2007; 20(5):449-459.

19. Nielson SJ, Popkin BM. Changes in beverage intake between 1977 and 2001. Am J Prev Med 2004; 27(3):205-210.

20. González CA, Jakszyn P, Pera G, Agudo A, Bingham S, Palli D, Ferrari P, Boeing H, del Giudice G, Plebani M, Carneiro F, Nesi G, Berrino F, Sacerdote C, Tumino R, Panico S, Berglund G, Simán H, Nyrén O, Hallmans G, Martinez C, Dorronsoro M, Barricarte A, Navarro C, Quirós JR, Allen N, Key TJ, Day NE, Linseisen J, Nagel G, Bergmann MM, Overvad K, Jensen MK, Tjonneland A, Olsen A, Bueno-de-Mesquita HB, Ocke M, Peeters PH, Numans ME, Clavel-Chapelon F, Boutron-Ruault MC, Trichopoulou A, Psaltopoulou T, Roukos D, Lund E, Hemon B, Kaaks R, Norat T, Riboli E.. Meat intake and risk of stomach and esophageal adenocarcinoma within the European Prospective Investigation into Cancer and Nutrition (EPIC). J Nat Cancer Center 2006; 98(5):345-354.

21. Jain MG, Rohan TE, Soskolne CL, Kreiger N. Calibration of the dietary questionnaire for the Canadian Study of Diet, Lifestyle and Health cohort. Publ Health Nutr 2003; 6(1):79-86.
22. Thompson FE, Kipnis V, Midthune D, Freedman LS, Carroll RJ, Subar AF, Brown CC, Butcher MS, Mouw T, Leitzmann M, Schatzkin A. Performance of a food-frequency questionnaire in the US NIHAARP (National Institutes of Health-American Association of Retired Persons) Diet and Health Study. Publ Health Nutr 2007; 11(2):183-195.

23. Bingham SA, Day NE, Luben R, Ferrari P, Slimani N, Norat T, Clavel-Chapelon F, Kesse E, Nieters A, Boeing $\mathrm{H}$, Tjønneland A, Overvad K, Martinez C, Dorronsoro M, Gonzalez CA, Key TJ, Trichopoulou A, Naska A, Vineis P, Tumino R, Krogh V, Bueno-deMesquita HB, Peeters PH, Berglund G, Hallmans G, Lund E, Skeie G, Kaaks R, Riboli E; European Prospective Investigation into Cancer and Nutrition. Dietary fiber in food and protection against colorectal cancer in the European Prospective Investigation into Cancer and Nutrition (EPIC): an observational study. Lancet 2003; 361(9368):1496-1501.

24. Ferrari P, Day NE, Boshuizen HC, Roddam A, Hoffmann K, Thiébaut A, Pera G, Overvad K, Lund E, Trichopoulou A, Tumino R, Gullberg B, Norat T, Slimani N, Kaaks R, Riboli E. The evaluation of the diet/disease relation into the EPIC study: considerations for the calibration and the disease models. Int J Epidemiol 2008; 37(2):368-378.

25. Fraser GE, Stram DO. Regression calibration in studies with correlated variables measured with error. Am J Epidemiol 2001; 154(9):836-844.

26. Kaaks R, Plummer M, Riboli E, Estève J, van Staveren W. Adjustment for bias due to errors in exposure assessments in multicenter cohort studies on diet and cancer: a calibration approach. Am J Clin Nutr 1994; 59(Suppl.1):245S-50S.

27. Plummer M, Clayton D. Measurement error in dietary assessment: an investigation using covariance structure models. Part. I. Stat Med 1993; 12(10):925935.

28. Kipnis V, Subar AF, Midthune D, Freedman LS, Ballard-Barbash R, Troiano RP, Bingham S, Schoeller DA, Schatzkin A, Carroll RJ. Structure of dietary measurement error: results of the OPEN biomarker study. Am J Epidemiol 2003; 158(1):14-21.

Artigo apresentado em 30/04/2010

Aprovado em 31/08/2010

Versão final apresentada em 09/09/2010 